\title{
Experimental verification of high spectral entanglement for pulsed waveguided spontaneous parametric down-conversion
}

\author{
Avenhaus, M.; Chekhova, M. V.; Krivitsky, Leonid; Leuchs, G.; Silberhorn, C.
}

Published in:

Physical Review A

Link to article, DOI:

10.1103/PhysRevA.79.043836

Publication date:

2009

Document Version

Publisher's PDF, also known as Version of record

Link back to DTU Orbit

Citation (APA):

Avenhaus, M., Chekhova, M. V., Krivitsky, L., Leuchs, G., \& Silberhorn, C. (2009). Experimental verification of high spectral entanglement for pulsed waveguided spontaneous parametric down-conversion. Physical Review A, 79(4), 043836. https://doi.org/10.1103/PhysRevA.79.043836

\section{General rights}

Copyright and moral rights for the publications made accessible in the public portal are retained by the authors and/or other copyright owners and it is a condition of accessing publications that users recognise and abide by the legal requirements associated with these rights.

- Users may download and print one copy of any publication from the public portal for the purpose of private study or research.

- You may not further distribute the material or use it for any profit-making activity or commercial gain

- You may freely distribute the URL identifying the publication in the public portal 


\title{
Experimental verification of high spectral entanglement for pulsed waveguided spontaneous parametric down-conversion
}

\author{
Malte Avenhaus, ${ }^{1}$ Maria V. Chekhova, ${ }^{1,2}$ Leonid A. Krivitsky, ${ }^{1,3,4}$ Gerd Leuchs, ${ }^{1}$ and Christine Silberhorn ${ }^{1}$ \\ ${ }^{1}$ Max Planck Institute for the Science of Light, Günther-Scharowsky-Straße 1/Bau 24, 91058 Erlangen, Germany \\ ${ }^{2}$ Department of Physics, M.V. Lomonosov Moscow State University, Leninskie Gory, 119992 Moscow, Russia \\ ${ }^{3}$ Department of Physics, Technical University of Denmark, Fisikvej 309, 2800 Lyngby, Denmark \\ ${ }^{4}$ Data Storage Institute, $A^{*}$ STAR, 5 Engineering Drive, 117608 Singapore, Singapore
}

(Received 28 November 2008; published 30 April 2009)

\begin{abstract}
We study the spectral properties of spontaneous parametric down-conversion (SPDC) in a periodically poled waveguided structure of potassium-titanyl-phosphate (KTP) crystal pumped by ultrashort pulses. Our theoretical analysis reveals a strongly entangled and asymmetric structure of the two-photon spectral amplitude for type-II SPDC. We confirm these predictions experimentally by measuring single-photon spectra, on one hand, and the dependence of Hong-Ou-Mandel interference visibility on the width of spectral filtering, on the other hand.
\end{abstract}

DOI: 10.1103/PhysRevA.79.043836

PACS number(s): 42.65.Lm, 42.50.Dv, 03.67.Bg, 42.50.Ex

Recently, entanglement of single photons in continuous variables, such as frequency and wave vector, became an important issue in quantum optics [1-6]. Various measures of entanglement are discussed in the literature in this connection, among them, the Schmidt number [1] and the operational measure introduced in [2]. While some research directions require single-mode biphotons (photon pairs weakly entangled in continuous variables) $[7,8]$, others are aimed at obtaining highly entangled biphoton light. Besides fundamental interest, attention on this subject is caused by the possibility of applying higher-dimensional Hilbert spaces in quantum information protocols such as, for instance, quantum key distribution [9]. It is especially promising to use continuous-variable entanglement together with polarization entanglement.

In this paper, we explore frequency entanglement for biphotons generated via type-II spontaneous parametric downconversion (SPDC) [10] in a periodically poled (PP) potassium-titanyl-phospate (KTP) waveguide from femtosecond pump pulses. Due to the high power density of the pump along the whole length of the structure, PP nonlinear waveguides can be extremely efficient sources of photon pairs [11-14]. At the same time, the large length of the waveguide in combination with the short pulse duration can be expected to create two-photon states highly entangled in frequency. In other words, the two-photon spectral amplitude (TPSA) should have an extremely stretched shape, leading to large widths of signal and idler single-photon (marginal) spectra but, at the same time, narrow spectral width of coincidences [15]. To verify this conclusion experimentally, the most direct method would be measuring the joint two-photon spectrum by simultaneously filtering and scanning the frequencies of signal and idler photons [3,5]. However, similar to its modification, based on Fourier spectroscopy [16], this method is not feasible here because it requires spectral filtering with bandwidth much less than the TPSA coincidence width (in our case, on the order of $0.02 \mathrm{~nm}$ ). In the case of extremely high entanglement considered here, such narrowband filtering would lead to a reduction in the coincidence counting rate by about 4 orders of magnitude and, hence, to an unacceptable increase in the measurement errors and/or acquisition times.

We test the entanglement structure of type-II SPDC generated in a PPKTP waveguide in a more indirect manner: we measure, on one hand, the single-photon spectra of signal and idler photons and, on the other hand, the visibility of the Hong-Ou-Mandel (HOM) interference [17] depending on the bandwidth of more broadband filtering, with full width at half maximum (FWHM) on the same order of magnitude as the TPSA width (about $0.2 \mathrm{~nm}$ ). The idea of this measurement is illustrated in Fig. 1, which shows schematically the square module of the TPSA, often called two-photon spectral intensity (TPSI). Due to the different group velocities of the signal and idler photons, the TPSA of type-II biphotons gen-

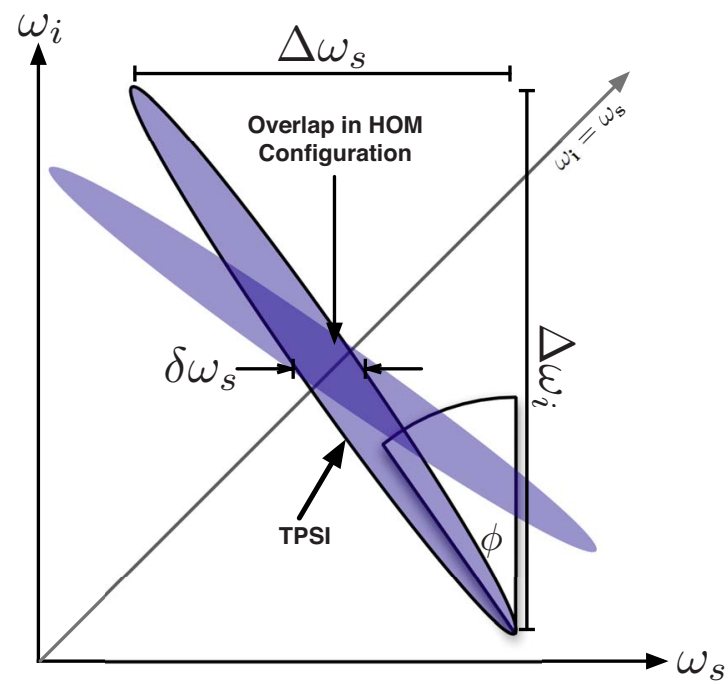

FIG. 1. (Color online) Schematic shape of the two-photon spectral intensity (TPSI). The HOM interference visibility depends on the relative overlap between TPSI and its reflection with respect to the $\omega_{i}=\omega_{s}$ line. A small relative overlap always indicates that TPSI is stretched, and then the TPSI tilt can be experimentally found from the widths of single-photon spectra $\Delta \omega_{s}, \Delta \omega_{i}$. By measuring the overlap versus the filtering bandwidth, one can find the TPSI aspect ratio. 


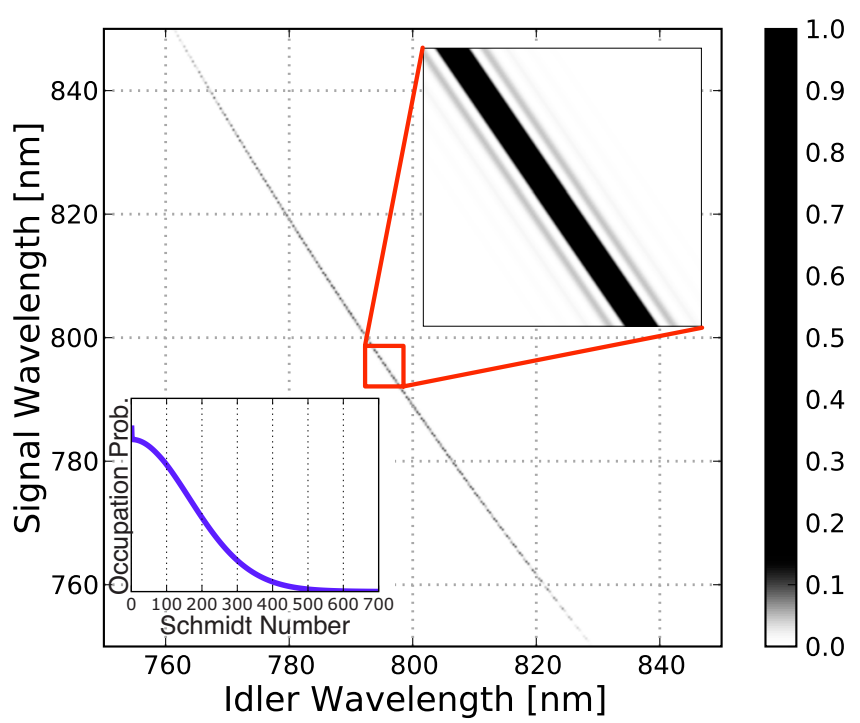

FIG. 2. (Color online) TPSI on a large scale and after a zoom into its center (top right inset). Bottom left inset shows the Schmidt decomposition.

erated from a pulsed pump is usually asymmetric with respect to the exchange of signal and idler frequencies $\omega_{s}, \omega_{i}$ [18]. The visibility of the HOM interference is given by the relative overlap between the TPSA and its transpose with respect to the exchange $\omega_{s} \leftrightarrow \omega_{i}$. It will then depend, first, on the "tilt" $\phi$ of TPSA and, second, on its aspect ratio $A$ (assume that $A>1)$. Note that $A$ and $\phi$ determine the operational measure of frequency entanglement introduced in [2] as the ratio of the TPSI "single-photon" spectral width to its "coincidence" width, $R \equiv \Delta \omega_{s} / \delta \omega_{s}$. If the overlap is small, it is evidence that $A \gg 1$, regardless of the tilt $\phi$, and then the tilt can be determined from the widths of single-photon spectra: $\tan \phi=\Delta \omega_{s} / \Delta \omega_{i}$. Filtering around the degeneracy point, even with a bandwidth comparable or larger than $\delta \omega_{s}, \delta \omega_{i}$, reduces $A$ and therefore increases the interference visibility. Hence, by measuring the visibility as a function of the filter bandwidth, one can find out the initial degree of entanglement, provided that the tilt $\phi$ is measured independently from the above considerations. Note that this method is only suitable for type-II biphotons.

Let us calculate the TPSA for type-II quasi-phasematched SPDC in a PPKTP waveguide pumped by femtosecond pulses. The waveguide propagation is along the $x$ axis, and the pump and idler waves are assumed to be $y$ polarized, while the signal wave is polarized along the $z$ axis. The two-photon state can be written in the form $[18,19]$

$$
|\Psi\rangle=\int d \omega_{s} d \omega_{i} F\left(\omega_{s}, \omega_{i}\right) a_{s}^{\dagger}\left(\omega_{s}\right) a_{i}^{\dagger}\left(\omega_{i}\right)|\mathrm{vac}\rangle,
$$

with the two-photon spectral amplitude $F\left(\omega_{s}, \omega_{i}\right)$ given by the product of the spectral amplitude $E_{p}(\omega)$ of the pump intensity and the "phase-matching function," determined by the properties of the nonlinear crystal,

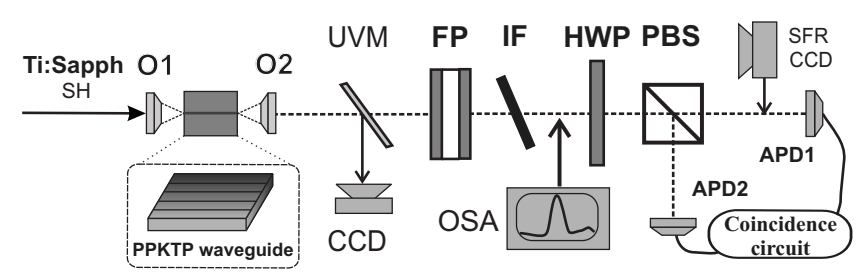

FIG. 3. Experimental setup: O1,2, coupling and decoupling objectives; UVM, highly reflective UV mirror; HWP, half-wave plate; PBS, polarizing beam splitter; APD1,2, single-photon counting modules (SPCMs); FP, Fabry-Pérot interferometer; OSA, optical spectrum analyzer; IF, interference filters; SPR CCD, singlephoton-resolving charge-coupled device (CCD) camera combined with a grating spectrometer.

$$
\begin{aligned}
F\left(\omega_{s}, \omega_{i}\right)= & E_{p}\left(\omega_{s}+\omega_{i}-\omega_{p}\right) \\
& \times \operatorname{sinc}\left[\frac{L}{2}\left\{k_{p}\left(\omega_{s}+\omega_{i}\right)-k_{s}\left(\omega_{s}\right)-k_{i}\left(\omega_{i}\right)-m K\right\}\right] .
\end{aligned}
$$

Here, $\omega_{p, s, i}$ are frequencies of the pump, signal, and idler radiation, respectively; $k_{p, s, i}$ are the corresponding wave vectors, $L$ is the length of the waveguide, and the "sinc function" is defined as $\operatorname{sinc}(x) \equiv \sin (x) / x$. The term $m K$ is due to the $m$ th-order quasi-phase-matching, with the inverse grating vector being related to the poling period $\Lambda$ as $K=2 \pi / \Lambda$. Because of the waveguide propagation, the transverse wavevector mismatch does not enter Eq. (2), and the pump, signal, and idler spatial modes are assumed to be the eigenmodes of the waveguide. Taking into account the dispersion dependence of bulk KTP as well as the waveguide-mode correction to the refractive indices [20], we calculated the TPSA for first-order quasi-phase-matching in a waveguide with $\Lambda=8.52 \mu \mathrm{m}$. As a function of signal and idler wavelengths, the TPSI is shown in Fig. 2. The pump was assumed to have a Gaussian spectrum, with the central wavelength of $397.65 \mathrm{~nm}$ and the width of $3.7 \mathrm{~nm}$.

From Fig. 2, showing the TPSI shape, and from the inset, zooming into its center, one can see that, first, the distribution is asymmetric with respect to the exchange of signal and idler wavelengths and, second, the state is highly entangled in frequency. Indeed, while the total widths of this distribution along signal and idler frequencies $\Delta \omega_{s}$ and $\Delta \omega_{i}$ are equivalent, respectively, to 60 and $40 \mathrm{~nm}$ (which agrees with the results of [14]), the spectral widths of coincidences are much narrower: 0.2 and $0.135 \mathrm{~nm}$. According to the definition in Ref. [2], this results in $R=300$. Calculation of the Schmidt number yields a very close figure of $N=301.5$ (the result of the Schmidt decomposition is shown in the inset of Fig. 2). Such a high degree of entanglement for a pulsed type-II source is due to the combination of a large crystal length, a broad pump spectrum, and the group-velocity dispersion [21].

The HOM interference visibility is determined by the "relative overlap" function [18], 


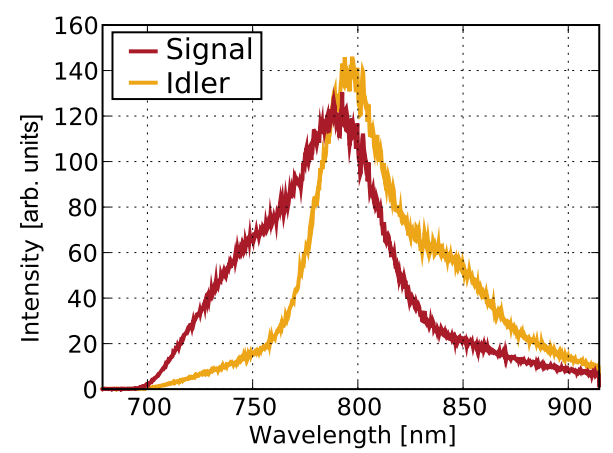

FIG. 4. (Color online) Single-photon spectra of signal and idler radiations.

$$
O \equiv \frac{\iint d \omega_{s} d \omega_{i} F\left(\omega_{s}, \omega_{i}\right) F^{*}\left(\omega_{i}, \omega_{s}\right)}{\iint d \omega_{s} d \omega_{i}\left|F\left(\omega_{s}, \omega_{i}\right)\right|^{2}}
$$

which reduces with the increase in $R$. By using filters of a sufficiently small bandwidth, inserted into both signal and idler channels, one can reduce the degree of entanglement and hence increase the overlap $O$, which, in its turn, leads to an increase in the visibility.

In HOM interference experiments with a nonpolarizing beam splitter (NPBS) followed by two polarizers [17], the visibility is equal to the overlap $O$ [18]. Here, we use a different scheme in which the NPBS is replaced by a polarizing beam splitter (PBS), and the coincidence counting rate is recorded versus the orientation of a half-wave plate (HWP) inserted before the PBS. In this case, one can show that the visibility is related to the overlap function as

$$
V=\frac{1+O}{3-O},
$$

varying from $1 / 3$ (the classical limit) to 1 as the overlap changes from 0 to 1 .

Thus, from the measured dependence of the interference visibility on the filter bandwidth, we are able to find the TPSI coincidence width and the degree of entanglement without the necessity of performing very challenging narrow-band spectral selection.

Our experimental setup is shown in Fig. 3. A Ti:sapphire laser with the central wavelength of $795 \mathrm{~nm}$, the pulse duration of $160 \mathrm{fs}$, and a repetition rate of $76 \mathrm{MHz}$ was frequency doubled and used to pump a 12-mm-long PPKTP chip. The pump was coupled into the waveguide by using a $20 \times$ achromatic microscope objective, mounted on a threedimensional micrometer stage. On the surface of the PPKTP chip (from AdVR Corp.), there were waveguide channels with the size of $4 \times 4 \mu \mathrm{m}^{2}$ with different poling periods. The one used in our experiments had $\Lambda=8.52 \mu \mathrm{m}$. A $20 \times$ achromatic objective at the end of the waveguide chip was used to collimate the SPDC beam. The remaining part of the pump beam was eliminated by a high-reflectance UV mirror, which at the same time had 99\% transmission for the SPDC central wavelength. For alignment purposes we also monitored the spatial mode of the reflected pump beam by a CCD camera.

Marginal distributions of the two-photon amplitude were measured with a single-photon-sensitive CCD camera (Andor Tech.) accompanied by a grating spectrometer with a standard resolution of $0.5 \mathrm{~nm}$ and gave the results shown in Fig. 4. The results are in good agreement with the calculation. Although the maxima of two marginal distributions were slightly shifted, we observed a region of their strong intersection, which we further considered as a point of frequency degeneracy.

Hong-Ou-Mandel interference was observed with a rotating HWP followed by a polarizing beam splitter and two fiber-coupled avalanche photodiodes (SPCM from PerkinElmer). The output pulses from the detectors were electronically time gated by a trigger signal from the laser and then fed into a coincidence detection setup with a resolution window of $5 \mathrm{~ns}$. By recording the coincidences versus the orientation of the HWP, we characterized the visibility of the HOM interference. This measurement was performed under different filtering conditions. Note that all our measurements were made without the temporal walkoff compensation. This is because such compensation, while being crucial in the case of cw pumping, is almost useless for ultrafast SPDC [22].
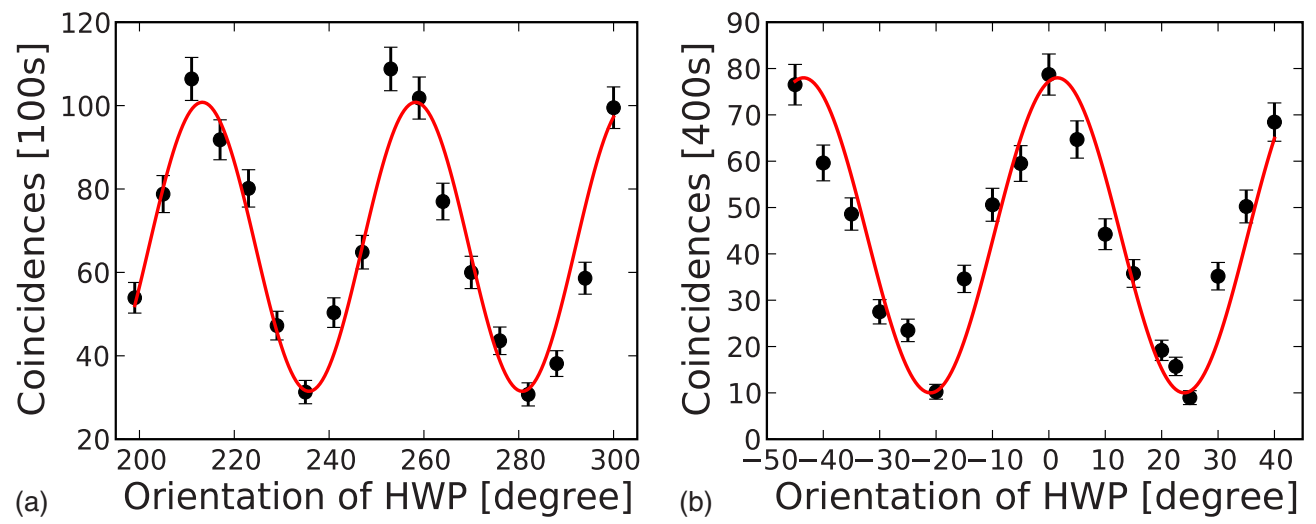

FIG. 5. (Color online) Experimental dependencies of the coincidence counting rate on the HWP orientation for the filtering bandwidths of (a) 0.45 and (b) $0.15 \mathrm{~nm}$. Solid lines show the fit of experimental data. 
In the absence of narrow-band filtering, we observed a visibility of $0.33 \pm 0.02$, which is the classical limit for our scheme. Spectral filtering performed only by an IF with $3 \mathrm{~nm}$ FWHM led to a visibility of $0.38 \pm 0.02$. In order to perform a strong spectral selection we installed a low-finesse $(\mathcal{F}=7)$ FP interferometer (LOMO PLC; insertion losses, $15 \mathrm{~dB}$ ), accompanied by a set of narrow-band IFs. We stress that the same spectral selection was performed for both signal and idler photons since the filter was placed before the PBS. In order to overcome the insertion losses introduced by the FP, we set the input mean pump power to $4 \mathrm{~mW}$ (in the measurements without the FP, the power was $1 \mathrm{~mW}$ ), which led to a parametric gain on the order of 0.1 and consequent noticeable contribution of accidental coincidences. This contribution was estimated as $R_{\mathrm{acc}}=R_{1} R_{2} / R_{\text {rep }}$, with $R_{1}$ and $R_{2}$ as the counting rates of the two detectors and $R_{\text {rep }}$ as the repetition rate of the pump. The rate of accidental coincidences was about 5-7 \% and was subtracted from the total coincidence counting rate.

By making the FP cavity length equal to $100 \mu \mathrm{m}$, we achieved a nearly Lorentzian transmission spectrum with width of $0.45 \mathrm{~nm}$. This spectrum was probed by passing a part of the fundamental bright beam directly through the filter and into an OSA with a resolution of $0.05 \mathrm{~nm}$. The other transmission bands of FP were suppressed by an IF with the FWHM of $3 \mathrm{~nm}$ and the central wavelength aligned, by means of a tilt, to match the SPDC degeneracy point. With this filtering, we obtained polarization interference with a visibility of $0.57 \pm 0.03$ [Fig. 5(a)]. By increasing the FP cavity length to $300 \mu \mathrm{m}$, we achieved a transmission spectrum with width of $0.15 \mathrm{~nm}$ (this required two differently tilted IFs for selecting a single transmission band), which resulted in a visibility of $0.79 \pm 0.03$ [Fig. 5(b)].

From the obtained values of interference visibility we calculated the "overlap function" [Eq. (4)], plotted in Fig. 6 versus the filtering bandwidth. In the same figure, the solid line shows the theoretical dependence calculated from Eqs. (2)-(4) for the parameters of our experiment. One can see that the experimental points follow the theoretical dependence, being about $15 \%$ below it. At first sight, this hints at a smaller coincidence width of TPSI. Indeed, Fig. 6 also shows the overlap dependence on the filter bandwidth calculated for the case of TPSI being twice as narrow (dotted line) and twice as broad (dashed line). However, a more likely explanation for the experimental points lying below the theoretical dependence is the nonideal overlap between the orthogonally polarized spatial modes of the waveguide. In fact, the total overlap function entering Eq. (4) is a product of the frequency overlap function and the corresponding spatial overlap, which does not depend on the spectral filtering. From the interference pattern observed for $0.15 \mathrm{~nm}$ filtering, we can conclude that the spatial overlap is not less than 0.73 . This

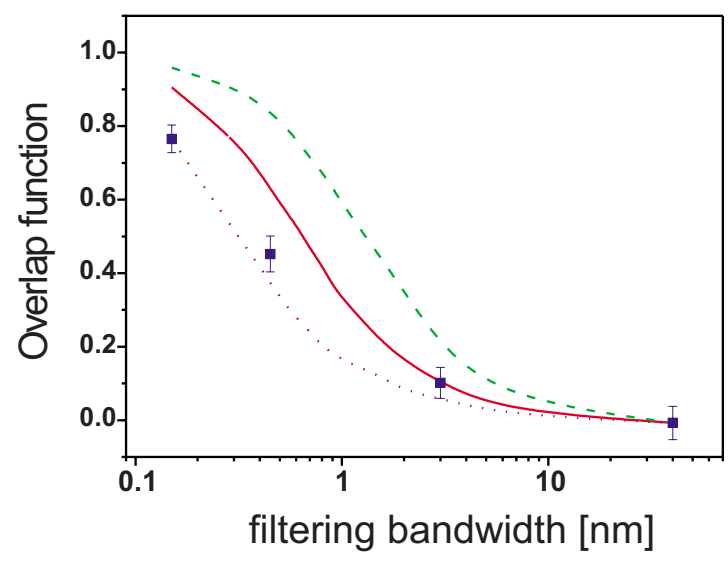

FIG. 6. (Color online) TPSA overlap measured (points) and calculated (solid line), versus the filter bandwidth. Dashed line shows the same dependence calculated for a two-photon state twice less entangled and dotted line shows that for a state twice more entangled.

allows us to find the upper bound for the TPSI coincidence width, or the lower bound for the degree of frequency entanglement achieved for waveguided type-II SPDC in PPKTP. Assuming the smallest possible spatial overlap to be 0.73 , we find that the operational measure of spectral entanglement is $R \geq 260$. At the same time, the upper bound for $R$ is given by the dotted line in Fig. 6 and yields $R \leq 600$.

In conclusion, we have calculated theoretically and probed experimentally the distribution of the two-photon spectral amplitude for light generated via type-II SPDC in a PPTKP waveguide in the case of femtosecond-pulsed pump. This distribution for a type-II source demonstrates a high (more than 260) degree of frequency entanglement. Our method of determining the degree of entanglement relies on filtering the spectrum of two-photon light and observing the dependence of HOM interference visibility on the selected spectral bandwidth. This method is preferable for highly entangled systems since it requires less narrow-band filtering than other methods $[3,16]$. HOM polarization interference has already been observed for cw-pumped SPDC in PPKTP waveguides [23]; our experiment is an observation of this type of interference in waveguides pumped by femtosecond pulses.

We acknowledge the financial support of the Future and Emerging Technologies (FET) program within the Seventh Framework Programme for Research of the European Commission, under the FET-Open grant agreement CORNER, Grant No. FP7-ICT-213681. L.A.K. acknowledges the support of Alexander von Humboldt foundation, and M.V.C., the support of the DFG foundation. 
[1] C. K. Law and J. H. Eberly, Phys. Rev. Lett. 92, 127903 (2004).

[2] M. V. Fedorov, M. A. Efremov, A. E. Kazakov, K. W. Chan, C. K. Law, and J. H. Eberly, Phys. Rev. A 69, 052117 (2004).

[3] Y.-H. Kim and W. P. Grice, Opt. Lett. 30, 908 (2005).

[4] A. B. U'Ren, R. K. Erdmann, M. de la Cruz-Gutierrez, and I. A. Walmsley, Phys. Rev. Lett. 97, 223602 (2006).

[5] H. S. Poh, C. Y. Lum, I. Marcikic, A. Lamas-Linares, and C. Kurtsiefer, Phys. Rev. A 75, 043816 (2007).

[6] M. V. Fedorov, M. A. Efremov, P. A. Volkov, E. V. Moreva, S. S. Straupe, and S. P. Kulik, Phys. Rev. Lett. 99, 063901 (2007).

[7] W. P. Grice, A. B. URen, and I. A. Walmsley, Phys. Rev. A 64, 063815 (2001).

[8] P. J. Mosley, J. S. Lundeen, B. J. Smith, P. Wasylczyk, A. B. U'Ren, C. Silberhorn, and I. A. Walmsley, Phys. Rev. Lett. 100, 133601 (2008).

[9] I. A. Khan and J. C. Howell, Phys. Rev. A 73, 031801(R) (2006); L. Zhang, Ch. Silberhorn, and I. A. Walmsley, Phys. Rev. Lett. 100, 110504 (2008).

[10] D. N. Klyshko, Photons and Nonlinear Optics (Gordon and Breach, New York, 1988).

[11] S. Tanzilli, Electron. Lett. 37, 26 (2001).

[12] K. Banaszek, A. B. U'Ren, and I. A. Walmsley, Opt. Lett. 26,
1367 (2001); A. Fedrizzi, T. Herbst, A. Poppe, and T. Jennewein, Opt. Express 15, 15377 (2007).

[13] C. E. Kuklewicz, M. Fiorentino, G. Messin, F. N. C. Wong, and J. H. Shapiro, Phys. Rev. A 69, 013807 (2004).

[14] A. B. U'Ren, C. Silberhorn, K. Banaszek, and I. A. Walmsley, Phys. Rev. Lett. 93, 093601 (2004).

[15] This width can be measured by selecting the signal (idler) frequency and measuring the coincidence counting rate versus the idler (signal) frequency.

[16] W. Wasilewski, P. Wasylczyk, P. Kelenderski, K. Banasek, and C. Radzewicz, Opt. Lett. 31, 1130 (2006).

[17] M. H. Rubin, D. N. Klyshko, Y. H. Shih, and A. V. Sergienko, Phys. Rev. A 50, 5122 (1994).

[18] W. P. Grice and I. A. Walmsley, Phys. Rev. A 56, 1627 (1997).

[19] T. E. Keller and M. H. Rubin, Phys. Rev. A 56, 1534 (1997).

[20] M. G. Roelofs, A. Suna, W. Bindloss, and J. D. Bierlein, J. Appl. Phys. 76, 4999 (1994).

[21] Yu. M. Mikhailova, P. A. Volkov, and M. V. Fedorov, Phys. Rev. A 78, 062327 (2008).

[22] Y. H. Kim, M. V. Chekhova, S. P. Kulik, M. H. Rubin, and Y. Shih, Phys. Rev. A 63, 062301 (2001).

[23] M. Fiorentino, S. M. Spillaine, R. G. Beausoleil, T. D. Roberts, P. Battle, and M. W. Munro, Opt. Express 15, 7479 (2007). 